\title{
CRITICAL SOUND ATTENUATION AND SPIN-LATTICE RELAXATION
}

\author{
A. Pawlak \\ Institute of Physics, A. Mickiewicz University \\ Matejki 48/49, 60-769 Poznań, Poland
}

The nonasymptotic critical properties of sound propagation are studied in compressible magnetic system. The role of coupling of order parameter and acoustic phonons to spin-energy density is investigated and the ultrasonic attenuation scaling function is found.

PACS numbers: $0.5 .70 . \mathrm{Jk}, 62.65 .+\mathrm{k}$

Analysis of sound propagation experiments in magnetic systems is complicated by the occurrence of several different regimes, depending on whether or not energy exchange between the spins and the phonon system is important at a frequency of interest. In the present paper we investigate a model where in addition to the coupling to two order parameter fluctuations [1,2] the sound mode couples linearly to the fluctuations of spin energy which relaxes with spin-lattice relaxation time $\tau_{\mathrm{SL}}$. In some materials (predominantly magnetic insulators) this mechanism can give rise to quite important contributions to dispersion and attenuation for some suitable values of $\tau_{\mathrm{SL}}$ [3]. Specifically, we discuss model described by the free energy functional

$$
\begin{aligned}
H= & \frac{1}{2} \int \mathrm{d}^{d} x\left\{r_{0} S^{2}+(\nabla S)^{2}+\tilde{u}_{0} S^{4}+C_{12}^{0}\left(\sum_{\alpha} e_{\alpha \alpha}\right)^{2}+2 C_{44}^{0} \sum_{\alpha, \beta} e_{\alpha \beta}^{2}\right. \\
& \left.+g_{0} \sum_{\alpha} e_{\alpha \alpha} S^{2}+f_{0} \varepsilon S^{2}+w_{0} \varepsilon \sum_{\alpha} e_{\alpha \alpha}+\frac{1}{C_{0}} \varepsilon^{2}\right\},
\end{aligned}
$$

where $S$ denotes the order parameter, $e_{\alpha \beta}$ is the strain tensor and $\varepsilon-$ spin-energy density.

The dynamics of our model is defined by the coupled Langevin equations for the order parameter $S_{k}$, acoustic phonons $Q_{k}$ and the spin-energy density $\varepsilon_{k}$ :

$$
\begin{aligned}
& \dot{S}_{k}=-\Gamma_{0} \frac{\partial H}{\partial S_{-k}}+\xi_{k}, \\
& \ddot{Q}_{k, \lambda}=-\frac{\partial H}{\partial Q_{-k, \lambda}}-D_{\lambda}^{0} k^{2} \dot{Q}_{k, \lambda}+\eta_{k, \lambda}, \\
& \dot{\varepsilon}_{k}=-\Lambda \frac{\partial H}{\partial \varepsilon_{-k}}+\Psi_{k} .
\end{aligned}
$$


Using a functional representation of the above equations one can find the acoustic self-energy

$$
\begin{aligned}
& \Sigma(k, \omega)=k^{2}\left\{w_{0}^{2} D_{0}(k, \omega)+2\left[g_{0}-w_{0} f_{0} D_{0}(k, \omega)\right]^{2}\right\} \\
& \quad \times\left\langle\Gamma_{0}(\tilde{S} S)_{k, \omega} S_{-k,-\omega}^{2}\right\rangle_{L_{\mathrm{OP}}^{\text {eff }}} .
\end{aligned}
$$

The effective order parameter Lagrangian $L_{\mathrm{OP}}^{\mathrm{eff}}$, used for calculation of the response function, contains phonon- and spin energy-mediated interactions whose effect can be represented by a shift of the parameter

$$
\tilde{u}_{0} \rightarrow u_{0}=\tilde{u}_{0}-k^{2} g_{0}^{2} G_{0}(k, \omega)-\left(f_{0}-g_{0} \omega_{0} G_{0}(k, \omega)\right)^{2} D_{0}(k, \omega)
$$

with $G_{0}^{-1}(k, \omega)=-\omega^{2}-\mathrm{i} D_{0}^{\lambda} k^{2} \omega+c_{0}^{2} k^{2}$ and $D_{0}^{-1}=-\mathrm{i} \omega / \Lambda+C_{0}^{-1}$ being the bare phonon- and energy-response functions, respectively.

Finally, the sound attenuation coefficient can be found as

$$
\begin{aligned}
& \alpha(\omega, t)=A\left(g_{0}-f_{0} w_{0} C_{0}\right)^{2} \omega^{2} \\
& \quad \times \frac{\left(1 / \Gamma_{0}\right) t^{-(z \nu+\alpha)} I(y) / y+\left(C_{0}^{2} / \Lambda\right) t^{-\alpha}\left[I^{2}(y)+R^{2}(y)\right]}{[1+x I(y)]^{2}+x^{2} R^{2}(y)},
\end{aligned}
$$

where $y=\left(\omega / \Gamma_{0}\right) t^{-z \nu}$ is the reduced frequency with $\left(1 / \Gamma_{0}\right) t^{-z \nu}$ being the order-parameter characteristic time and $\mathrm{t}$ - the reduced temperature. $I(y)$ and $R(y)$ are scaling functions which, to the leading order, are given by $I(y)=\operatorname{Im} \Phi(y) / y$ and $R(y)=\operatorname{Re} \Phi(y)$ with

$$
\begin{aligned}
\Phi(y) & =\left[1+\left(\frac{y}{2}\right)^{2}\right]^{-\alpha / 4 \nu} \\
& \times\left\{1+\frac{\mathrm{i} \alpha}{y \nu}\left[\mathrm{i}\left(1-\frac{\mathrm{i} y}{2}\right) \operatorname{arctg}\left(\frac{y}{2}\right)-\frac{1}{2} \ln \left(1+\left(\frac{y}{2}\right)^{2}\right)\right]\right\} .
\end{aligned}
$$

In Eq.(5), $x=\omega\left(C_{0} / \Lambda\right) t^{\alpha}$ is a second reduced frequency which appears as a result of the fact that the spin energy-lattice relaxation time $\tau_{\mathrm{SL}}$ has two divergent components

$$
\tau_{\mathrm{SL}}=\tau_{\mathrm{SL}}^{0} t^{-\alpha}+\frac{B}{\Gamma_{0}} t^{-z \nu},
$$

where $\tau_{\mathrm{SL}}^{0}=C_{0} / \Lambda$ is the bare spin-lattice relaxation time and $B=\mathrm{O}\left(f_{0}^{2}\right)$ is a constant. Although asymptotically $\tau_{\mathrm{SL}}$ is proportional to the order parameter relaxation time $\tau_{\mathrm{OP}}=t^{-z \nu} / \Gamma_{0}$ (showing a strong divergence as $T \rightarrow T_{\mathrm{c}}$ ) the first term in Eq. (7) (which diverges only as specific heat) may be dominant within experimentally accessible temperature region if $\Lambda / C_{0} \ll \Gamma_{0}$.

Depending on the relative size of $\tau_{\mathrm{SL}}^{0}, \Gamma_{0}^{-1}, \omega$ and $t$, Eq. (5) shows two limiting behaviours. If the first term in Eq. (7) dominates one obtains small critical attenuation exponent equal to $2 \alpha$ in accordance with the phenomenological theory results (the case of the acoustic phonons coupled only to the energy) $[4,5]$ which assumed simple relaxational form of the energy response function. If the second term in Eq. (7) dominates we recover results of Iro and Schwabl [6] with strong singularity described by large sound attenuation exponent equal to $z \nu+\alpha$. It gives 
the asymptotic behaviour but in some materials it cannot be observed within the measured temperature range.

It should be mentioned that Eq. (5) is valid only in the "weak-coupling" limit $[2,7]$, i.e. for small $g_{0}$ and $w_{0}$. In the "strong-coupling" regime both values for the critical attenuation exponent should be lowered by $\alpha / 2$.

\section{References}

[1] R. Dengler, F. Schwabl, Z. Phys. B 69, 327 (1987).

[2] A. Pawlak, J. Phys., Condens. Matter 1, 7989 (1989).

[3] B. Lüthi, T.J. Moran, R.J. Polina, J. Phys. Chem. Solids 31, 1741 (1970).

[4] K. Kawasaki, in: Phase Transitions and Critical Phenomena, Vol. 5a, Eds. C. Domb, M.S. Green, Academic Press, New York 1976.

[5] B. Lüthi, in: Dynamical Properties of Solids, Vol. 3, Eds. G.K. Horton, A.A. Maradudin, North-Holland, Amsterdam 1980.

[6] H. Iro, F. Schwabl, Solid State Commun. 46, 205 (1983).

[7] A. Pawlak, Phys. Rev. B 44, 5296 (1991). 